\title{
Non-ideal explosive performance in a building structure
}

\author{
K. Kim ${ }^{1}$, W. Wilson ${ }^{1}$, J. Colon ${ }^{1}$, T. Kreitinger ${ }^{1}$, C. Needham ${ }^{2}$,

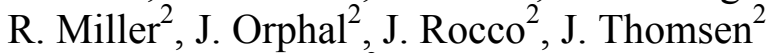 \\ \& L. V. Benningfield ${ }^{2}$ \\ ${ }^{I}$ Defense Threat Reduction Agency, USA \\ ${ }^{2}$ Applied Research Associates, Inc., USA
}

\begin{abstract}
The performance of a non-ideal explosive has been investigated in a reinforced concrete 2-room test structure with two different window and door configurations: (1) without and (2) with doors and a window. Pressure records at different locations of the two-room test structure for the two different configurations have been compared with each other and 3D CFD numerical simulations. Reaction efficiencies of fuel gases and aluminum particles in the explosive detonation products, especially the aerobic reaction with air, as functions of time have been estimated for the two cases and then compared with each other.
\end{abstract}

Keywords: non-ideal explosives, aluminum particles, aerobic reaction, anaerobic reaction, tests, CFD simulations, payload-structure interaction.

\section{Introduction}

Generic non-ideal explosives are fuel-rich in detonation reactions. Much of the fuel, in the form of aluminum particles and some detonation products, will react with other detonation products during an anaerobic reaction phase in a fireball. However, typically there is some leftover fuel that has a potential to react further with the surrounding air (aerobic reaction) at a later time. If it does, in what timescale does it react? Does it add to the strength of blast waves inside a structure? Would it work if the structure is open to the outside (doors and widows are removed from their openings)? Inside the structure, how much blast enhancement can be obtained if the structure is marginally and temporarily 
contained by responding doors and windows? If so, how much and through what mechanisms are these enhancements realized? These are the questions to be addressed by this paper.

Two identical tests, with the exception of the presence or absence of responding doors and windows, were conducted and then numerical simulations were performed to aid in determining the reasons for differences between the two cases, if any.

\section{Test description and results}

Test Charges: Each test charge was cylindrical having a diameter of $11.0 \mathrm{~cm}$, a length of $24.1 \mathrm{~cm}$, and a nominal mass of $3.73 \mathrm{~kg}$. The mild steel cases had a thickness of $0.625 \mathrm{~cm}$ on both the cylindrical portion and the end caps. The end caps were bolted to the cylindrical portion of the case resulting in a nominal total case mass of $5.45 \mathrm{~kg}$. The charges were end detonated with a detonator and a $50 \mathrm{~g}$ cylindrical high explosive booster $(3.175 \mathrm{~cm}$ diameter $\mathrm{x} 3.62 \mathrm{~cm}$ length) embedded in the main charge.

\subsection{Test-bed configuration}

The reinforced concrete test-bed configuration is shown in Figure 1. This 2-room configuration is one half of a 4-room test structure with the other half of the structure being separated from the two rooms used in these tests using steel non-responding doors (sealed hallways shown in black in Figure 1). The walls are $0.91 \mathrm{~m}$ thick steel reinforced concrete and do not deform during tests. The rooms have nominal $3.66 \times 4.57 \times 2.44 \mathrm{~m}$ inside dimensions. The windows are nominally $1.07 \mathrm{~m}$ wide by $0.91 \mathrm{~m}$ tall, and doors are nominally $1.07 \mathrm{~m}$ wide by $2.13 \mathrm{~m}$ tall. The charges were placed parallel to floor and parallel to the interior wall as indicated in Figure 1. The center-of-mass of each charge was located $1.09 \mathrm{~m}$ from the interior wall, $0.91 \mathrm{~m}$ from the floor, and $0.91 \mathrm{~m}$ from the wall opposite the window. The charges were end detonated from the end facing the window wall (bottom end in Figure 1). The source room interior was clad with $1.27 \mathrm{~cm}$ thick steel with an additional $1.27 \mathrm{~cm}$ steel cladding placed on the floor, walls, and ceiling in the fragment pattern of the test devices.

There were many gages employed inside and outside of the structure (see [1] for further details), but in this paper they are not shown. One of the static pressure gages is located in the middle of the source room in the ceiling (red triangle in Figure 1) and there are five other wall-mounted gages in the same room (blue squares in Figure 1, and two of them are located at position A at different heights). The gage selected for illustration in this paper is indicated as position " $\mathrm{A}$ " in the diagram and is located $76 \mathrm{~cm}$ from the floor in the corner of the source room opposite the corner where the charge was placed. This gage location is typical for pressure measurements inside the source room and has been documented to produce impulse readings within $+/-3 \%$ for a number of shots with identical charges. In one test (Test 1), all doors and windows were removed from the structure (leaving the structure open to the outside), and in the 
other (Test 2), two plywood doors and a plastic-film-covered window were placed as shown in Figure 1.

For Test 2, a $1.3 \mathrm{~cm}$ thick plywood door, slightly smaller than the steel opening (by $\sim 0.3 \mathrm{~cm}$ ), was mounted in the exterior doorway flush with the interior wall using three hinges on the left (as viewed from the interior). The right side of the door was secured with screws to three $5.1 \times 10.2 \times 7.6 \mathrm{~cm}$ wood blocks epoxied to the inside of the door frame. A $1.3 \mathrm{~cm}$ plywood door between the source and adjacent rooms was flush with the inside of the adjacent room and was mounted with hinges and wood blocks as for the source-room door (hinges on the left as viewed from the adjacent room).

For Test 2, the window opening was fitted with standard $0.64 \mathrm{~cm}$ thick glass with a window film on the surface facing out of the structure. The surface of the glass was set just outside the inner surface of the room, mounted in a $5.1 \mathrm{x}$ $10.2 \mathrm{~cm}$ wooden frame. The wood frame was supported from behind by $2.54 \mathrm{~cm}$ angle steel tack-welded to the window opening. After the test, the steel was still in place but the wood frame had been ejected.

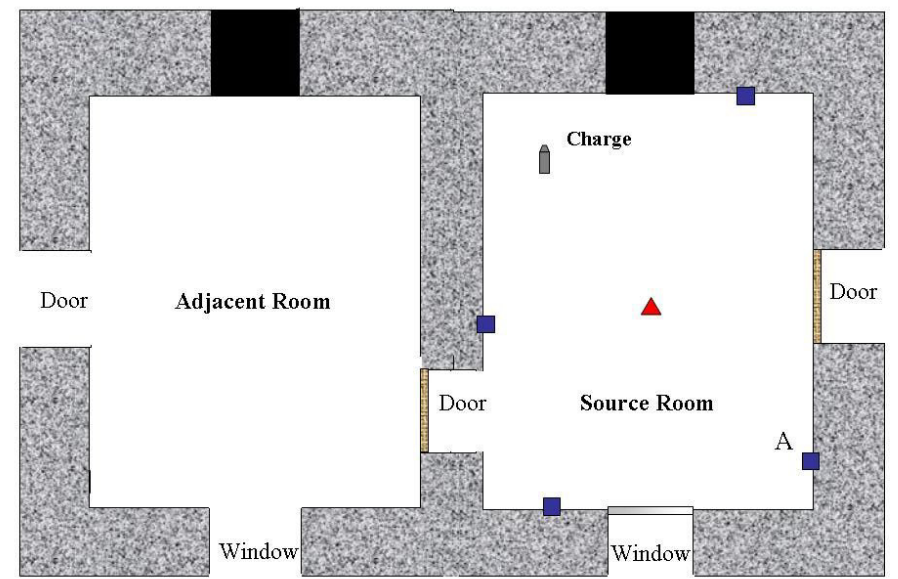

Figure 1: Test bed configuration and gage locations. Shown with doors and window in place (Test 2 configuration).

\subsection{Test results}

Figure 2 shows the comparisons of typical pressure records taken for the two test configurations. The solid blue lines show the static pressure for Test 1 with unrestricted openings, and the broken red lines show the same for Test 2 where the doors and window were in place. From the video results, it was concluded that the plywood doors were ejected as intact units within a few ms after the impact of the first blast wave. The behaviour of the window was not clear, but the mass of the glass probably provided a temporary confinement effect within the structure. It is this temporary containment effect by the doors and window that influence the explosive performance in the structure. 


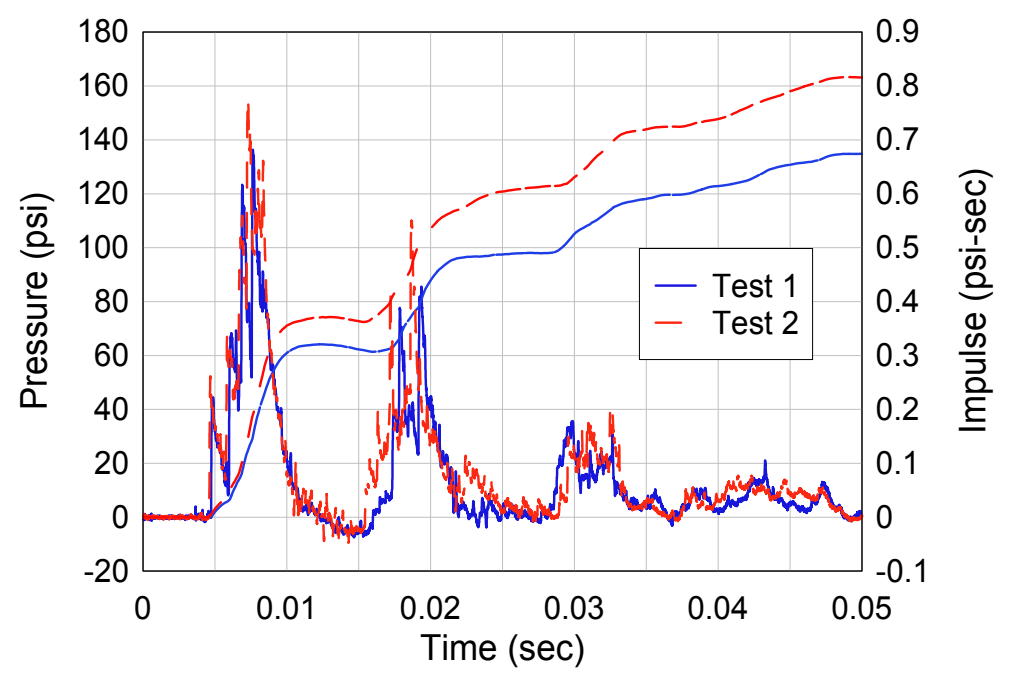

Figure 2: $\quad$ Pressure and impulse records for Test 1 (solid blue) and Test 2 (dashed red).

Qualitatively, the two tests exhibited similar behaviours, showing similar peaks and valleys. There were several shock wave vibrations in the room due to geometric effects within the first ten to twenty ms. The first pressure peaks were almost the same in magnitude for both cases, as they should be since the first peak travels directly from the detonation of the charge to the sensor without being influenced by the structure surfaces. Subsequent peaks are reflected waves and some reflected secondary waves can be larger than the first waves.

A major difference between the two tests is that in the case where doors and the window are in place (Test 2), the pressure does not decay as fast as the case where they are absent (Test 1). This can be clearly seen in the impulse curve comparisons. In the source room wall locations, the difference is about $15-20 \%$ in impulse. In the adjacent room, although not shown here, most of the gages show a difference of about $10-20 \%$ in impulse.

The difference of $15-20 \%$ in impulses may not seem significant, especially if one examines the peak pressure only. A non-ideal explosive is not expected to show as much difference since the majority of aluminum fuel particles are expected to react in anaerobic reactions within the detonation product gases, and only a small portion of aluminum particles are expected to react with the surrounding air. Although the difference is small, it is well outside the experimental error and is useful in understanding the phenomenology involved. Also, this difference is directly related to the kinetic energy of secondary debris in conventional deformable structures and can play a very important role.

The main question is: Is this difference coming from a more localized distribution (because of the slight confinement effect of the doors and the window) of the same total energy from the detonation of the charge? Or, does 
the temporary confinement effect of the doors and the window affect the reaction efficiency of the charge as well? To be more precise, does the temporary confinement enhance the aerobic and anaerobic reaction efficiencies of a non-ideal explosive and therefore its total energy output? How long does the confinement effect last? From the pressure data, it might be conjectured that there must be some residual reaction going on within the structure. In order to gain some insight into answering these questions, numerical calculations were made simulating the two test cases.

\section{Numerical simulations with SHAMRC}

\subsection{The CFD model}

The SHAMRC ${ }^{2}$ CFD non-ideal explosives model attempts to incorporate first-principles physics and chemistry models into a CFD code. The goal of this model is to predict post-detonation environments for non-ideal explosives given the target geometry, weapon specifications, and explosive properties of the formulation. The model allows the detonation products and embedded aluminum particles to evolve over time, with the particles being heated and cooled by the surrounding gasses and accelerated by the drag of the blast flow gases within the target geometry. If the temperature of the aluminum particles reaches $2050 \mathrm{~K}$, and if there is oxygen present nearby, the aluminum particles are allowed to ignite and release energy. The reactions considered by the model are a "first-order" approach to capturing the post-detonation energy release of non-ideal explosives.

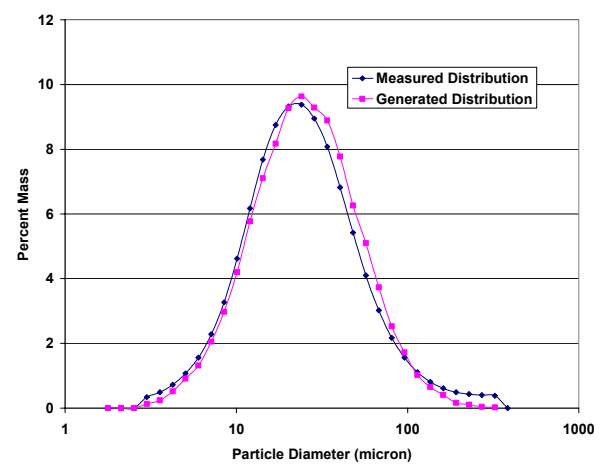

Figure 3: Aluminum particle size distribution.

In the model, aluminum particles, perhaps the most important fuel present in many non-ideal explosives, are treated as being spherical with the same surface-to-volume ratio as the actual particles. The model approximates the size distribution used in a particular explosive mixture. For example, Figure 3 shows a comparison between the MDX-81 particle size distribution used in some aluminized explosives and the distribution generated by the model. The size 
distribution of aluminum particles is important because the heating, and therefore the ignition and burning of the aluminum particles are strongly dependent on the particle size.

The amount of carbon, water, methane, and "other burned explosive materials" released during the passage of the detonation front are estimated using the CHEETAH program provided by Lawrence Livermore National Laboratory. After the detonation products are released, the model allows them to react with atmospheric oxygen and aluminum particles. The following reactions are considered:

$$
\begin{aligned}
2 \mathrm{Al}+3 \mathrm{H}_{2} \mathrm{O} & \rightarrow \mathrm{Al}_{2} \mathrm{O}_{3}+3 \mathrm{H}_{2} \\
4 \mathrm{Al}+3 \mathrm{O}_{2} & \rightarrow 2 \mathrm{Al}_{2} \mathrm{O}_{3} \\
\mathrm{C}+\mathrm{O}_{2} & \rightarrow \mathrm{CO}_{2} \\
2 \mathrm{H}_{2}+\mathrm{O}_{2} & \rightarrow 2 \mathrm{H}_{2} \mathrm{O} \\
\mathrm{CH}_{4}+2 \mathrm{O}_{2} & \rightarrow 2 \mathrm{H}_{2} \mathrm{O}+\mathrm{CO}_{2}
\end{aligned}
$$

These equations represent the estimated primary reactions that occur after detonation and account for most of the post-detonation energy released based on this model. $\mathrm{CO}_{2}$ in the detonation products is not allowed to react while water vapour is. It should be noted CHEETAH predicts that hydrogen gas is not found in abundance after the detonation of a typical organic explosive, thus hydrogen gas is only available for combustion after aluminum has reacted with water. The model allows the hydrogen, released by the reaction of water with aluminum, to react with atmospheric oxygen.

In the SHAMRC calculations, the doors and window were represented as high density fluids with their density equal to that of the doors and windows. No material strength was given to small latches, screws, mounts, or door and window materials in the calculation. This approximation has been used for "responding" objects in past calculations with relatively accurate results. The doors and window of the structure may have been propelled out of the structure as single units, at least for the first few ms. However, it was deemed that any error from this "dense fluid" approximation would not alter any conclusions of the paper qualitatively.

Figures 4 and 5 show the comparison between measured and calculated pressures at gage location A. There are some differences between the calculations and the measurements. The calculated arrival time of the first peak is slow by $0.6 \mathrm{~ms}$ (in the figures, the calculated values are shifted by $0.6 \mathrm{~ms}$ ) and the calculated second major peak is also slower. The error may come from inaccurate description of initial detonation. Subsequent smaller peaks are somewhat out of phase and these differences may be due to the imprecise treatment of doors and windows. Figure 4 (Test 1) shows better comparison between measured and calculated pressures than Figure 5 (Test 2). It is conjectured that, in Test 2, hinges, mounts and such that were ignored in the calculation may play some role in holding the confinement slightly higher, and therefore the measured values are somewhat higher than the calculated. 


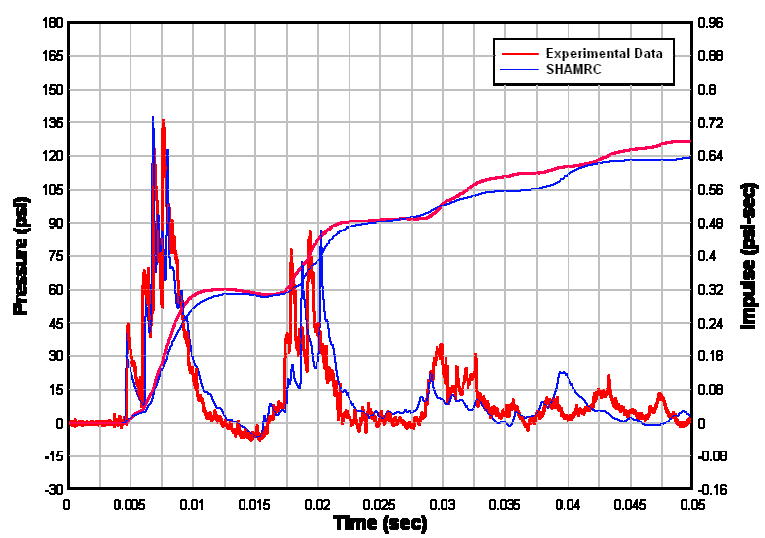

Figure 4: Measured and calculated pressures for Test 1 (unrestricted openings).

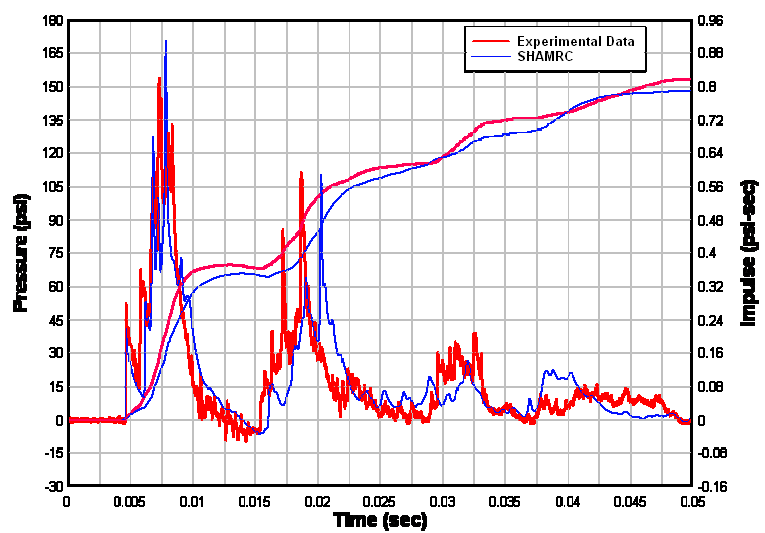

Figure 5: $\quad$ Measured and calculated pressures for Test 2 (doors and window in place).

However, the overall comparison of Figures 4 and 5 is very good and this type of good correlation has been demonstrated for other non-ideal explosives and thermobarics in a number of other tests. It is therefore assumed that the SHAMRC model is emulating most of the physics and chemistry fairly well even though it uses a fairly small set of chemical reactions and uses the "heavy fluid" approximation for responding objects.

\subsection{Combustion efficiency of aluminum particles}

One possibility for the increase in pressure due to the extra confinement provided by the responding doors and window is aluminum combustion efficiency increase for the non-ideal explosive. Other reactions are assumed to 
occur as soon as mixing (with air) takes place whereas the aluminum reaction additionally requires that the particle temperature reaches its ignition temperature. Since the confinement increases the temperature inside the structure, aluminum reaction will benefit from the confinement, but not others. Figures 6 and 7 illustrate the combustion efficiency of aluminum predicted by the model for the two test conditions including the contributions of aerobic and anaerobic combustion of aluminum.

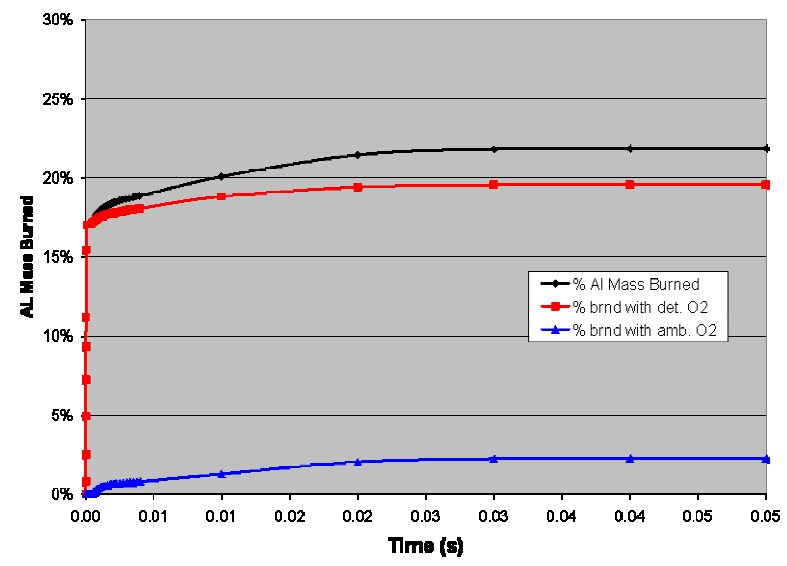

Figure 6: Aluminum combustion for Test 1 (unrestricted openings).

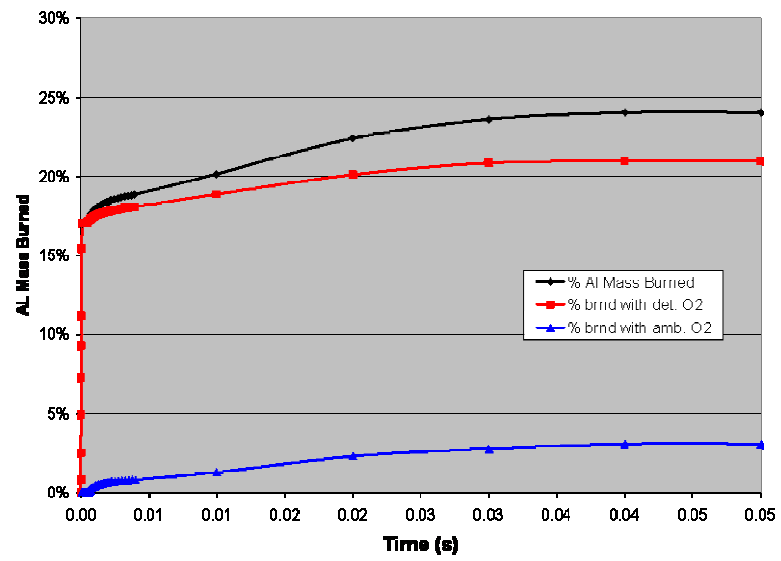

Figure 7: $\quad$ Measured and calculated pressures for Test 2 (doors and window in place). 
An interesting observation drawn from Figures 6 and 7 is that much of the aluminum does not burn. The total amount of aluminum reacted is only about $22-24 \%$ (black lines). Of this, about $17 \%$ reacts very quickly (the nearly vertical line near time zero) after the detonation in an anaerobic reaction. The anaerobic reaction afterwards accounts only for 2 or $4 \%$ respectively and the aerobic reaction accounts only for 2 or $3 \%$ respectively, depending on the two test configurations.

The total aluminum reaction is increased only by $2 \%$ by the marginal confinement for this explosive. Enhancement of total energy release (not shown in the paper), is likewise increased by about $2 \%$ only. A large amount of post detonation reaction actually comes from carbon reaction (not shown) for this explosive. Most of the post detonation reaction for carbon is completed by about $30 \mathrm{~ms}$, indicating that mixing with air takes that amount of time.

The aerobic reaction enhancement (the lower blue lines in Figures 6 and 7) is slightly less than that of the anaerobic reaction (the middle red lines). The fact that only a small amount of aluminum reacts at all indicates that aluminum does not get mixed with air in a short enough time when the temperature is high. Late time anaerobic reaction as well as aerobic reaction of aluminum takes about 30 ms (Figures 6 and 7). After $30 \mathrm{~ms}$, the temperature of the air is no longer high enough to ignite the aluminum particles. A large amount of available energy stored in the explosive, therefore, is wasted during these events. Even some of the energy released by other fuel such as carbon may not contribute significantly to the enhancement of useful impulse, if they are released at later times when pressure peaks gets smaller.

Although not shown in the paper, the model predicts the door to the exterior door to begin moving at about $8 \mathrm{~ms}$ and is well outside the structure in about $20 \mathrm{~ms}$, which correlates fairly well with the video data.

\section{Conclusions}

It has been demonstrated that the performance of non-ideal explosives (pressure, impulse and reaction efficiency) depends on target configurations. CFD calculations that closely approximate the measured pressure profiles indicate that the aluminum reaction efficiency is slightly enhanced by a temporary and marginal confinement such as responding doors and windows. This model also indicates that much of the aluminum in the explosive does not burn in these configurations. One reason may be attributed to limited mixing of aluminum particles with the ambient air at early times. Another reason may be the very high ignition temperature $(2050 \mathrm{~K})$ of the aluminum particles.

\section{References}

[1] J. E. Colón, G. Sofran, W. Wilson, S. Babcock, L.V. Benningfield, Michael Kaneshige, Enhanced Blast Standardized Test Program, SAVIAC 75th Symposium Proceedings, Virginia Beach, VA, 2004. 
524 Structures Under Shock and Impact IX

[2] Watry, C., Needham, C., Perry, J., Schneider, J. (2004). Thermobaric ACTD Phenomenology and Explosive Model Development Report - Phase II. Applied Research Associates, Albuquerque, New Mexico. 